This paper is a preprint of a paper submitted to and accepted for publication in IET Microwaves Antennas and Propagation and is subject to Institution of Engineering and Technology Copyright. The copy of record is available at IET Digital Library. Some final publisher edits and corrections may not be included in this file.

\title{
Frequency Selective Fading of Ultrawideband Wireless Channels in Confined Environments
}

Vit Sipal ${ }^{1}$, Javier Gelabert ${ }^{1}$, Ben Allen ${ }^{2}$, Christopher Stevens ${ }^{1}$, David Edwards ${ }^{1}$

${ }^{1}$ Dept. of Engineering Science, University of Oxford

2 Department of Computer Science \& Technology, University of Bedfordshire

\section{Abstract}

As a result of the recent ruling by the European Commission, ultrawideband wireless communications can now pervade small confined environments such as cars or trains. This paper studies frequency selective fading experienced by ultrawideband wireless channels in these confined environments. It focuses on the relationship between the severity of fading and the environment size using an analytical modelling, simulation and measurement. We show that the frequency selective fading is more severe in smaller environments because the bandwidth required to resolve all multipaths in time is inversely proportional to the cubic root of the volume of the environment. We show that the ECMA International standards for ultrawideband proposed by the WiMedia alliance may not provide sufficient means of mitigating the issues associated with the frequency selective fading in confined environments such as cars.

\section{Introduction:}

Currently, short range ultrawideband (UWB) wireless systems are being deployed as real products, for example as wireless audio-visual links for streaming media in indoor environments such as in the office or the living room [1],[2]. In the future, short range wideband wireless systems can be expected to be deployed in more confined environments such as aircraft, road vehicles and even electronic enclosures such as computers. For instance, the current European regulation for UWB (from 2009) implemented changes to allow UWB deployment inside automotive and railway vehicles [3]. Research has been reported investigating and assessing the potential of wireless UWB communication among computer components [4]. One of the basic issues of such small confined environments is the fact that their wave propagation properties are fundamentally different due to the high number of multipath components. Wave propagation in confined environments, and UWB in particular, has been studied quite extensively. For instance, measurements were conducted in cars [5]-[8], tunnels and mine galleries [9]-[10], or even inside computer cases [4]. This paper focuses on the issue of frequency selective fading and especially on the general dependency of fading as a 
function on size of the environment. To the best of our knowledge, such a study of UWB channels has not yet been conducted.

In wireless communications, fading represents the main contributor towards frequency selectivity. Fading is defined as the local spatial variation of channel energy and is caused by the superposition of multipath components at the receiver [11]. In most applications, it is desirable that the data rate should not change for small movements of the transmitter or receiver [12], and as a result, the system must have means to mitigate the impact of fading. Even though fading is a well known phenomenon, the relationship between its severity and the size of the environment has not yet been studied.

In real situations, the manifestation of fading appears as a minimum in the transfer function of the wireless channel. A popular way to manage the impact of fading and frequency selectivity is to include a fade margin into link budget so that, even in the case of a minimum in the channel transfer function, the receiver still has an acceptable signal-to-noise ratio. References [13]-[17] have shown that fading is less severe for wideband systems largely due to the fact that, for larger bandwidths, individual multipaths can be resolved and the destructive superposition of multipaths is less likely. In other words, the required fade margin is significantly reduced when the bandwidth of the transmitted signal increases. Thus UWB wireless systems are inherently more robust to frequency selective fading than narrowband transmission. This effect is called fade depth scaling and was acknowledged to be present in a car environment [5].

One solution to reduce the impact of fading is the use of spreading the information signal over a frequency band. For example, in the ECMA International standard supported by the WiMedia alliance which uses OFDM, one information bit is modulated onto several carriers [18]. Such a technique improves performance through exploiting the increased frequency diversity and also helps to protect against the frequency selectivity of the channel.

The WiMedia standard adopted by ECMA International is intended to work in indoor environments such as rooms, offices etc. Previous work has shown that real systems fail to provide the maximum data rate of $480 \mathrm{Mbps}$ in a small van [6].

The authors of [7] estimated, that with the ECMAs bandwidth of $528 \mathrm{MHz}$, a UWB system operating at $200 \mathrm{Mbps}$ inside a car requires the path loss lower than approx. $50 \mathrm{~dB}$. According to [7] and [8], the standard path loss for in-car transmissions for a distance larger than $1 \mathrm{~m}$ (dashboard to rear seats) is $48.8 \mathrm{~dB}$ for an empty car and $52 \mathrm{~dB}$ for an occupied car. These values correspond to the data reported in [5]. The path loss is at the limit of the maximum path loss that allows a data rate of $200 \mathrm{Mbps}$ using the ECMA standard. If fading is taken into account, it is apparent that the ECMA system would not be able to provide full coverage of a standard car at the data rate of $200 \mathrm{Mbps}$. 
This illustrates the need for quantification of the relation between severity of fading and size of the environment to determine the size limit. This facilitates the adoption of current standards or to the development of new standards applicable to such environments.

The structure of this paper is as follows. The fading is first investigated by the means of an analytical channel model, and the change of the fading's impact with the size of the environment is thus quantified. The previously published fade-depth scaling model is compared to the measures for elimination of fading effects in the WiMedia ECMA standard. Ray-tracer simulations for 21,200 different confined environments were conducted to quantify the severity of fading effects as a function of the size of the environment. The ray tracing simulation is compared to previously published experimental data and data from additional measurements conducted in two confined environments with volumes of $3 \mathrm{~m}^{3}$ and $0.3 \mathrm{~m}^{3}$ in the frequency range $3-20 \mathrm{GHz}$. Then, parameters describing fading obtained from the simulation are compared to the measures the ECMA International standard applies to overcome fading so that the suitability of the standard for UWB wireless operating in confined environments can be evaluated.

\section{Fading in confined environments}

\subsection{Analytical model of fading}

The investigation starts with the channel impulse response model as proposed by the IEEE 802.15.4a model [21]:

$h(\tau)=\sum_{l=1}^{L_{c}} \sum_{k=1}^{L_{r}} \alpha_{k, l} e^{j \varphi_{k, l}} \delta\left(\tau-T_{l}-\tau_{k, l}\right)$

where $h(\tau)$ is the channel impulse response, $\alpha_{k, l}$ and $\varphi_{k, l}$ represent the multipath gain and phase coefficient of the $k$ th ray in $l$ th cluster, $T_{l}$ stands for the time-of-arrival of $l$ th cluster, $\tau_{k, l}$ is ray arrival time within individual cluster, $\delta(t)$ is Dirac impulse.

This can be easily converted to frequency domain using Fourier transformation.

$H(f)=\sum_{l=1}^{L_{c}} \sum_{k=1}^{L_{r}} \alpha_{k, l} e^{-j\left(2 \pi f\left(T_{l}+\tau_{k, l}\right)-\varphi_{k, l}\right)}$

The UWB channel power can then be calculated in the frequency domain by integration over a bandwidth $B W$ around centre frequency $f_{0}$ :

$\int_{f_{0}-B W / 2}^{f_{0}+B W / 2}|H(f)|^{2} d f$

The evaluation of the integral has been conducted in [22], here we use the same approach but we correct few minor mistakes of [22] regarding the signs of phases $\varphi_{m, n}, \varphi_{k, l}$ and definition of $B_{l, n}^{k, m}$ : 


$$
\begin{aligned}
\int_{f_{0}-B W / 2}^{f_{0}+B W / 2}|H(f)|^{2} d f & \\
= & B W \sum_{l=1}^{L_{c}} \sum_{k=1}^{L_{r}} \alpha_{k, l}^{2} \\
& +\frac{1}{2 \pi} \sum_{l=1}^{L_{c}} \sum_{\{=1}^{L_{c}} \sum_{k=1}^{L_{r}} \sum_{m=1}^{L_{r}} \frac{\alpha_{k, l} \alpha_{m, n}}{B_{l, n}^{k, n}} \\
& \times\left(\sin \left(2 \pi\left(f_{0}+\frac{B W}{2}\right) B_{l, n}^{k, m}+\varphi_{m, n}-\varphi_{k, l}\right)\right. \\
& \left.-\sin \left(2 \pi\left(f_{0}-\frac{B W}{2}\right) B_{l, n}^{k, m}+\varphi_{m, n}-\varphi_{k, l}\right)\right)
\end{aligned}
$$

where $B_{l, n}^{k, m}=\left(T_{l}+\tau_{k, l}\right)-\left(T_{n}+\tau_{m, n}\right)$ is the time difference between rays from different clusters , and $\{k, l\} \neq\{n, m\}$ represent the summation condition $l \neq n$ OR $k \neq m$

The result from [22] is unsuitable for evaluating of the impact of the environment's dimension on fading. Thus, the subtraction of the sin functions in the last bracket is performed:

$$
\begin{aligned}
\int_{f_{0}-B W / 2}^{f_{0}+B W / 2}|H(f)|^{2} d f & \\
= & B W \sum_{l=1}^{L_{c}} \sum_{k=1}^{L_{r}} \alpha_{k, l}^{2} \\
& +\frac{1}{\pi} \sum_{l=1}^{L_{c}} \sum_{n=1}^{L_{c}} \sum_{\{l, k\} \neq\{n\}}^{L_{r}} \sum_{m=1}^{L_{r}} \frac{\alpha_{k, l} \alpha_{m, n}}{B_{l, n}^{k, m}} \sin \left(\pi B W B_{l, n}^{k, m}\right) \cos \left(2 \pi f_{0} B_{l, n}^{k, m}+\varphi_{m, n}-\varphi_{k, l}\right) \\
& =B W\left(\sum_{l=1}^{L_{c}} \sum_{k=1}^{L_{r}} \alpha_{k, l}^{2}\right. \\
& +\sum_{l=1}^{L_{c}} \sum_{n=1}^{L_{c}} \sum_{\substack{k=1 \\
\{l, k\} \neq\{, m\}}}^{L_{r}} \sum_{m=1}^{L_{r}} \alpha_{k, l} \alpha_{m, n} \operatorname{sinc}\left(\pi B W B_{l, n}^{k, m}\right) \cos \left(2 \pi f_{0} B_{l, n}^{k, m}+\varphi_{m, n}-\varphi_{k, l}\right)
\end{aligned}
$$

Eq. (5) provides a useful insight into the nature of fading. From the final form of formula (5), the physical reason for fading can be seen. For a displacement of the transmitter or the receiver, the delay, $B_{l, n}^{k, m}$, between two rays from two different clusters will change. According to (5), this has an impact on the path loss because the value of the cos- and sinc-term changes. Changes of the 
coefficients $\alpha_{k, l}, \alpha_{m, n}$ will not be considered because, by definition, fading is a random variation of path loss for small displacements. These displacements are small enough not to represent a significant variation of the path loss. Thus, as far as the fading is concerned, $\alpha_{k, l}, \alpha_{m, n}$ are approximately constant and their effect is negligible compared to the impact of delay $B_{l, n}^{k, m}$.

The impact of bandwidth on the severity of fading, as reported in [13]-[17], can be observed. In the final form of Eq. (5), there are two main terms. The double summation is independent of bandwidth. The second quadruple summation, with the $\cos$ - and sinc-term, depends on bandwidth and as the bandwidth is increased, its contribution to the total sum is reduced with the amplitude of the sinc function. This is exactly the effect of fade depth scaling described in [13]-[17]. Moreover, if bandwidth is very small (i.e., the product $\left(\pi B_{l, n}^{k, m} B W\right)$ approaches zero), the value of the sincfunction approaches the value 1 . Therefore, there is an upper bound for channel power variation imposed by the gain coefficients and possible values of the cos-term. Thus, the fade depth is also bounded from above, as presented by experimental results in $[16,17]$.

The effect of confined environments such as trains, cars or the inside of computer consoles can be observed. In such environments, the average delay between the rays is reduced due to the smaller dimensions of the environment, therefore, it takes a larger bandwidth to mitigate the fading. Whilst it is apparent the effect of fading is negligible for infinite bandwidth, it is useful to know the relationship between the minimum bandwidth required to limit the fading below a selected level and the size of the room:

$B W_{\min k, l, m, n} \cdot B_{l, n}^{k, m}>A$

Where $A$ is a constant which can be specified depending of the required level of suppression of the sinc function.

For a specific known channel Eq. (6) can be resolved for all rays. For a general statement, the $E\left\{B_{l, n}^{k, m}\right\}$ must be evaluated. Two types of delays enter $B_{l, n}^{k, m}:$ the delays of clusters $T_{n}, T_{l}$, and the delays of individual rays within the cluster $\tau_{k, l}, \tau_{m, n}$. The intra-cluster delays have been shown in [23] to represent the manifestation of the antenna impulse response. Reference [24] then showed that the waveform of this manifestation is independent of small displacement of receiver or transmitter. Whilst these delays have impact on frequency selectivity, this frequency selectivity has different cause than fading, it is independent on the room volume and it does not change for small antenna displacements. Therefore, only the impact of inter-cluster delays $T_{n}, T_{l}$ has any impact on fading.

From Eq. (6) it is apparent that the mean delay between consecutive clusters $E\left\{B_{l, l+1}^{k, m}\right\}$ will be the most important in terms of an impact on the severity of fading because they will define the largest minimum bandwidth. The impact of $\tau_{k, l}, \tau_{m, n}$ is neglected as explained above, thus the relevant 
term is $\left\{B_{l, l+1}\right\}$, which is the mean delay between consecutive clusters. From a simple geometrical consideration, the mean delay between consecutive cluster will increase linearly with the size of the room, or if related to the volume, $V$, of the room, the dependency can be expected to be proportional to $V^{\frac{1}{3}}$. As a result, it can be concluded that the bandwidth required to resolve all multipath is inversely proportional to cubic root of the volume of the environment, i.e.,

$B W_{\min } \propto \frac{1}{E\left\{B_{l, l+1}\right\}} \propto V^{-\frac{1}{3}}$

To conclude, the analytical model of fading confirms that, in confined environments, the effect of fading will be more severe than in usual indoor environments.

\subsection{Fade mitigation performance}

Fade depth scaling relates the variation of the channel energy to the signal bandwidth. This variation is known to reduce for larger bandwidths. References [14]-[17] define fade depth as three times the standard deviation of channel energy for a small spatial variation of the receiver/transmitter. If this value is included as a fade margin to the link budget, in more than $99 \%$ of the cases the receiver energy will be sufficient. The fading is frequency selective, thus [13]-[17] suggest that wideband systems will be more robust against fading than their narrowband counterparts.

The ECMA Standard for UWB, which specifies OFDM as the chosen waveform together with punctured convolution coding, has three modes of frequency diversity [18]. In the first mode, with the lowest bit-rate, each bit is modulated at four different sub-carrier frequencies. This means an increase of transmitted energy by $6 \mathrm{~dB}$. In the second mode, each bit is modulated on two subcarrier frequencies giving an increase of $3 \mathrm{~dB}$. In the third mode, that is not obligatory in implementation, the bits are modulated on one sub-carrier frequency only. The third mode provides the highest bit-rate but it also is the most vulnerable mode to fading.

To compare with experimental data of fade-depth scaling presented in [12] at the bandwidth of $500 \mathrm{MHz}$ (closely corresponding to the $528 \mathrm{MHz}$ bandwidth of an OFDM block in ECMA standard [18]), the fade depth is determined to be $4 \mathrm{~dB}$. Measurements from [17] show a variation of 3-5 dB. These values are below the $6 \mathrm{~dB}$ level that is used by the first mode of the ECMA standard indicating that the fade-depth as described in [14]-[17] is a good mean for estimating the required frequency spreading factor for a system.

\subsection{Case study - ray tracing and measurements}

To prove the hypothesis that frequency selective fading becomes more severe in confined environments and therefore that existing wireless standards may not be sufficient in such scenarios, extensive ray tracing simulations of confined environments were conducted and compared to real measurement data.

\subsubsection{Ray tracing setup}


21,200 environments were modelled, which were generated as follows. Each environment consisted of a rectangular box. The dimensions of the box were given as: $\operatorname{rand}_{1} \cdot b \times \operatorname{rand}_{2} \cdot b \times b$, where rand $_{i}$ are random variables with uniform distribution from the interval $\langle 1 ; 2\rangle$. In total, 200 different pairs of $\operatorname{rand}_{1}$ and rand $_{2}$ were generated. The length parameter, $b$, was swept in 106 steps from $0.5 \mathrm{~m}$ to $30 \mathrm{~m}$. The steps were logarithmically distributed.

The reflection coefficient of the walls was assumed to be - 0.9. This value was estimated using the assumption of a perfect electric conductor (reflection -1) with losses counting for the thin layer of fabric/plastic as a cover of the metal in car interiors. The attenuation was estimated using attenuation constants of materials presented in [11]. For the sake of simplicity, the reflection coefficient was assumed to be constant for the whole UWB range. In real scenarios, this might not be the case but this simplification significantly reduces the computational effort with no significant impact on the results as the path loss is dominant over the reflection losses. Rays with up to six reflections were considered. The ray tracer uses the tile algorithm, which uses copies of the rectangular room to determine the impulse response of the channel in the time domain. The ray tracing algorithm is described in more detail in [19].

For the case of modelling cars, which have a significant proportion of windows, it should be noted that a different model for the reflection coefficient is required in order to produce meaningful results. However, modern higher class vehicles already have metallic foils in windows to protect against heat [20]. It can be anticipated that such windows will soon appear in middle and lower class vehicles too. Moreover, according to [6] even standard glass represents a noticeable reflector. The exact value of the reflection coefficient is not as important because, for high reflection coefficients (modulus $>0.8$ ), the power of each ray is dominated by the path loss associated with it. Therefore, an approximation of the reflection coefficient is sufficient.

Another concern may be that real cars are not shaped as rectangular boxes, but as presented in the theoretical analytical model, the important factor is the delay between consecutive ray clusters. Intuitively, these delays depend mainly on the volume of the environment rather than on the actual shape. The fading severity is therefore believed to depend mainly on the room volume. Objects inside the car such as seats or people introduce additional propagation effects such as shadowing and additional reflections. These effects will be discussed in the conclusion.

The position of the transmitter and receiver was selected as follows. The transmitter was placed at position $[0.25 b ; 0.3 b ; 0.6 b]$. The receiver location was varied in a grid of $20 \times 20$ points spaced by $1 \mathrm{~cm}$. The square was in the horizontal xy-plane. The centre of the grid was at $[0.75 b ; 0.7 b ; 0.2 b]$. The inter-element spacing is approximately $\lambda / 2$ for the highest frequency that was used, i.e., 12.75 $\mathrm{GHz}(\lambda=2.35 \mathrm{~cm})$. This frequency corresponds to a centre frequency of $7.25 \mathrm{GHz}$ and total bandwidth 
of $11 \mathrm{GHz}$. The channel transfer functions were determined for the frequencies $1-16 \mathrm{GHz}$, then the fade depth scaling with bandwidth was determined at centre frequency of $7.25 \mathrm{GHz}$ as in [14]-[17]. 7.25 GHz was chosen as it is the centre frequency of the UWB band 6- $8.5 \mathrm{GHz}$, which is the only UWB band in Europe that can be utilised without detect-and-avoid algorithms or low duty-cycle mode of operation.

The calculation of fade depth is performed as follows. The channel power for a selected bandwidth is calculated using Eq. (3) for each position of the receiver. The fade depth is defined as three times the standard deviation of the path loss within the local area [14]-[17]. The local area is defined by maximum displacement of $4 \mathrm{~cm}$ in both direction, thus 81 measurement points. This calculation represents one data point in Fig. 1 and is therefore repeated for a range of bandwidths $1 \mathrm{MHz}-12$ $\mathrm{GHz}$ swept logarithmically. This range is selected to enable characterization of the fade depth in both narrowband and wideband channels. The fade depth results for several rooms characterised by their volume are presented in Fig. 1. It must be noted that the selection of the local area is essential. It cannot be too large because while movement by $4 \mathrm{~cm}$ is negligible for $b$ larger than $2 \mathrm{~m}$ in terms of path loss of the direct path, it is significant for the smallest, i.e., $b=0.5 \mathrm{~m}$. On the other hand, if the area is too small, it will not contain the full variation of the path loss caused by the multipath superposition.

As can be seen in Fig. 1 for room dimensions in the order that would correspond to a car $(b=1.5 \mathrm{~m})$ , the fade depth at $0.5 \mathrm{GHz}$ bandwidth corresponding to the ECMA UWB standard is approximately 7 $\mathrm{dB}$. This is more than the $6 \mathrm{~dB}$ energy margin used by the ECMA in mode 1 and this suggests that even the highest protection against fading introduced in current standards may not be sufficient for systems operating in confined environments. Therefore, the results suggest that for confined environments such as automotive vehicles or computer enclosures, a new standard with increased fade margins will have to be developed to mitigate the issue of frequency selective fading.

\subsubsection{Measurement setup}

To further support the results of the ray tracing simulations, a number of real measurements were conducted in three different environments. The first are indoor measurements in a room with a volume of $55 \mathrm{~m}^{3}$ and are the same set of measurements as reported in [17]. The other two measurement sets were conducted in the same way as the measurements in [17] (employing the $\mathrm{XY}$-positioning table and a vector network analyzer) but they were conducted in confined environments with volumes of $3 \mathrm{~m}^{3}(1.75 \mathrm{~m} \times 1.65 \mathrm{~m} \times 1.05 \mathrm{~m})$ in Fig. $\left.3 \mathrm{a}\right)$, and $0.3 \mathrm{~m}^{3}(0.75 \mathrm{~m} \times 0.66$ $\mathrm{m} \times 0.58 \mathrm{~m})$ in Figs. 2 and 3 . The environments were built using a wooden frame covered with aluminium foil. In both cases, the positioning table was also covered with aluminium foil so that all the walls are the same. Discone antennas were $0.3 \mathrm{~m}$ above the positioning table. $\mathrm{ln}$ the first 
measurement set there was a space between the table and the wooden frame so that cables can reach the transmitter and receiver antennas. In the second setup, Fig. 3, the aluminium foil of the vertical walls did not reach all the way to the table making a space for the cable and the optical fibre. However, the leakage from these slots can be neglected because the measurement used discone antennas which radiate most of their energy into the upper hemisphere of their radiation pattern. The fade depth scaling for a centre frequency of $7.25 \mathrm{GHz}$ was investigated in the same way as in [17] and as summarised in section 2.3.1.

The measurement used bandwidths of $3-20 \mathrm{GHz}$. Lower bound was determined by the lower cutoff frequency of the discone antennas. The upper limit was given by the bandwidth of the available wideband amplifier. At such high frequencies the cable losses are significant. Thus, a Radio-overFibre link was used as a cable replacement. During the measurement the vector analyzer was calibrated to a reference 'through' connection that replaced the antennas and the channel. The transmitter was swept in a grid with a spacing of $6 \mathrm{~mm}$ (slightly less than $\lambda / 2$ at $20 \mathrm{GHz}$ ). The size of the grid was $40 \times 40$ points for the room and $3 \mathrm{~m}^{3}$ environment and it was $30 \times 30$ points for the 0.3 $\mathrm{m}^{3}$ due to limited space available. The position of both antennas is highlighted in the top view in Figs. 2 and 3.

\subsubsection{Results}

The model for fade depth scaling presented in [17] was used to study the dependencies on the room size in more detail. For the sake of clarity, the model from [17] is summarised. The fade depth scaling with bandwidth can be modelled with the following formula:

$F D=A+\frac{B}{1+C \cdot B W}$

$B W$ is for the variable bandwidth; $A, B$, and $C$ are model parameters.

The model and the meaning of parameters is presented in Fig. 4., using data taken from [17], which shows the measured fade depth as a function of bandwidth and the fitted curve according to the model from Eq. (6). The parameter $A$ represents the minimum fade depth for infinite bandwidth. The second parameter $B$ describes the difference in fade depth between narrowband and wideband channels. It can be understood as a penalty that narrowband channels have to pay in the form of fade margin that must be included in the link budget. Reference [17] presents another important parameter: $1 \mathrm{~dB}$ breakpoint bandwidth. This is the bandwidth at which the fade depth increases $1 \mathrm{~dB}$ above the minimum level given by parameter $A$ (see Fig. 4). It defines the bandwidth above which the channel behaves as a wideband channel with minimum influence of fading.

$B P(1 \mathrm{~dB})=\frac{B-1}{C}$

The resulting dependency of the minimum fade depth on the volume of a room as determined by simulation and measurement is presented in Fig. 5. There are two curves in Fig. 5. The grey curve 
represents the values obtained for all the 21,200 rooms sorted by their volume. The thick black line is the best fit of the data based on minimum mean square error (MMSE) fitting. This fit is used to emphasize the trend of the data. The equation of the fit is:

$$
A=0.96 V^{-0.171}
$$

The increase of the minimum fade depth may be attributed to the fact that for the smallest room, the fixed local area of 81 points already contains the impact of path loss dependency on distance whereas this dependency is negligible for larger rooms.

The ray tracing model results vary because every fade depth scaling curve is random since the fading is given by the relative position of the transmitter and receiver to the walls and other reflectors which is random. Parameter $A$ is used to quantify a random curve; hence it is a random variable also with mean and variance. From Fig. 5, the minimum level of fading increases as the room volume is decreased and the increase is exponential.

Fig. 5 enables the simulated data to be compared to the experimental data. There is one data-point for the $0.3 \mathrm{~m}^{3}$ and $3 \mathrm{~m}^{3}$ environments and 2 data-points for the measurement in room taken from [17]. In [17], 77 sets of measurements (7 distances and 11 different centre frequencies) were presented in a room with a volume of approx. $55 \mathrm{~m}^{3}$. The measured parameter, $A$, is determined to be below $1 \mathrm{~dB}$ for 62 out of 77 measurements while the maximum value was $1.5 \mathrm{~dB}$ which corresponds to the spread in Fig. 5 . Fig. 5 presents only the 2 data-points related to the centre frequency of $7.25 \mathrm{GHz}$. The slight differences between the measurement and the simulation results can be explained by the use of ideal isotropic antennas for the simulations and real discone antennas for the measurement. Thus, some multipaths, e.g. floor reflections, are significantly weaker for real measurements.

The other model parameter is $B$. Its dependency on room volume is presented in Fig. 6 . Apart from slight deviations from the trend for very small rooms (volumes below $1 \mathrm{~m}^{3}$ ), this parameter is almost independent on the room volume with dependency given by:

$$
B=12.9 V^{-0.029}
$$

The slight increase can be explained by the relatively large modulus of $\alpha_{k, l}$ in the analytical model (Eq. (1)-(5)) for smaller environments as mentioned in section 2.

The dependency of the breakpoint bandwidth, $B P(1 \mathrm{~dB})$, on the size of the room is presented in Fig. 7. It can be seen that the bandwidth required for minimising the effects of fading significantly increases for confined environments. Again, a good agreement between simulation and measurement is observed. This data corresponds to the data for different centre frequencies from [17]. The trend of the minimum mean square fit (MMSE) is given as:

$$
B P(1 d B)=2.50 V^{-0.295}
$$


Which corresponds well to the initial model expectation of minimum bandwidth being scaled proportionally to $V^{-0.33}$.

\subsection{Importance for real systems}

The ray tracing simulations show a good agreement with the experimental data from [17] and they confirm that the fading is more severe for smaller environments. Hence, such environments are more challenging for fade mitigation measures. The comparison in section 2.2 shows that the parameters of fade mitigation measures in the ECMA standard ( $0 \mathrm{~dB}$ to $6 \mathrm{~dB})$ are selected to deal with fading in indoor environments where fade depth for bandwidth of about $500 \mathrm{MHz}$ is on average $4 \mathrm{~dB}$. As shown above, the fade depth is higher of confined environment for the same bandwidth. Thus, the measures for mitigating the impact of fading can be expected to be insufficient for confined environment, which was confirmed by tests in [6]. Moreover, in real confined environments such as a car, the effect will be amplified because seats and people introduce shadowing, which causes an additional reduction of SNR for all OFDM carriers [7]. In other words, shadowing also increases the likelihood of bit errors which further strains forward error correction employed by the ECMA standard.

\section{Conclusion}

Frequency selective fading increases the probability of error in wireless transmission. To enable operation in the presence of frequency selective fading, wireless systems include measures to overcome it. This paper has explored the issue of frequency selective fading in confined environments.

The analytical model predicts that in small confined environments, such as interiors of cars or inside computer consoles, frequency selective fading will be a more severe issue. The model predicts that the bandwidth required to resolve all multipaths is scaled proportionally to $V^{-0.33}$ where $V$ is the volume of the environment. Ray tracing simulations predict scaling proportional to $V^{-0.295}$. This result compares well to the experimentally gathered data.

A simple comparison of the experimentally determined fade depth and the measures against fading included in the ECMA UWB standard [14] show that in confined environments the measures included in the ECMA standard are likely to fail to protect the data against frequency selective fading. As a result, it is suggested that systems for confined environments such as cars, trains, airplanes or inside of a computer console will have to use enhanced measures to overcome frequency selectivity (higher fade margins, wider bandwidth, more diversity, more coding or a combination). The exact specification of such codes will require further intensive measurement campaigns to characterise those new environments. Alternatively, the environments could be engineered to reduce the severity of fading, e.g., by the use of absorber walls. 



\section{References:}

[1] Mlinarsky, F.; Ziegler, J.; “Comprehensive UWB product testing: Part 1 - Part 3" EE Times, Dec. 2007

[2] Mlinarsky, F.; "Wireless HD video: Raising the UWB throughput bar (again)" EE Times, Feb. 2008 [3] "Decision to make the Wireless Telegraphy (Ultra-Wideband Equipment) (Exemption) Regulations 2009", Ofcom, 2009

[4] Gelabert, J.; Kavatjikidis, A.; Edwards, D.J.; Stevens, C.J.; "Experimental UWB channel characterisation of an electromagnetically small environment," Antennas \& Propagation Conference, 2009. LAPC 2009. Loughborough , vol., no., pp.381-384, 16-17 Nov. 2009

[5] Kobayashi, T.; , "Measurements and Characterization of Ultra Wideband Propagation Channels in a Passenger-Car Compartment," Spread Spectrum Techniques and Applications, 2006 IEEE Ninth International Symposium on , vol., no., pp.228-232, 28-31 Aug. 2006

[6] Garcia Zuazola, I.J.; Elmirghani, J.M.H.; Batchelor, J.C.; , "High-speed ultra-wide band in-car wireless channel measurements," Communications, IET , vol.3, no.7, pp.1115-1123, July 2009 [7] Schack, M.; Geise, R.; Schmidt, I.; Piesiewiczk, R.; Kurner, T.; "UWB channel measurements inside different car types," Antennas and Propagation, 2009. EuCAP 2009. 3rd European Conference on, vol., no., pp.640-644, 23-27 March 2009

[8] Schack, M.; Jemai, J.; Piesiewicz, R.; Geise, R.; Schmidt, I.; Kurner, T.; , "Measurements and Analysis of an In-Car UWB Channel," Vehicular Technology Conference, 2008. VTC Spring 2008. IEEE , vol., no., pp.459-463, 11-14 May 2008

[9] Lienard, M.; Degauque, P.; , "Natural wave propagation in mine environments," Antennas and Propagation, IEEE Transactions on , vol.48, no.9, pp.1326-1339, Sep 2000

[10] Chiba, J.; Inaba, T.; Kuwamoto, Y.; Banno, O.; Sato, R.; , "Radio Communication in Tunnels," Microwave Theory and Techniques, IEEE Transactions on , vol.26, no.6, pp. 439-443, Jun 1978

[11] B. Allen, M. Dohler, E. Okon, W. Malik, A. Brown, and D. Edwards, Ultra Wideband Antennas and Propagation for Communications, Radar and Imaging. John Wiley \& Sons, 2006

[12] H. Al-Raweshidy, "Optical fibre technologies and radio over fibre strategic research for future networks," eMobility Technology Platform EWG-4, White Paper, 2010

[13] Romme, J.; Kull, B.; "On the relation between bandwidth and robustness of indoor UWB communication," Ultra Wideband Systems and Technologies, 2003 IEEE Conference on , pp. 255- 259, 16-19 Nov. 2003

[14] Malik, W.Q.; Allen, B.; Edwards, D.J.; "Fade depth scaling with channel bandwidth," Electronics Letters, vol.43, no.24, pp.1371-1372, Nov. 222007

[15] Malik, W.Q., Allen, B., Edwards, D.J.; "Bandwidth-dependent modelling of smallscale fade depth in wireless channels," Microwaves, Antennas \& Propagation, IET, vol.2, no.6, pp.519-528, Sept. 2008 [16] Sipal, V.; Allen, B.; Edwards, D.J.; "Enhanced Fade Depth Model for Extremely Wideband Channels," Modelling and Simulation 2010, AMSE Conference on, June 2010, Prague [17] Sipal, V.; Allen, B.; Edwards, D.J.; "Exploration and Analysis of Fade Depth Scaling," Antennas \& Propagation Conference, 2010. LAPC 2010, November 2010, Loughborough [18] G. Heidari, WiMedia UWB - technology choice for wireless USB and Bluetooth. John Wiley \& Sons, 2008.

[19] Kavatjikidis, A.: "Measurement Analysis and Capacity Performance of Ultra-Wideband Radio Propagation Channels". PhD. Thesis, University of Oxford, Sep 2008 
[20] Kapagerof, C.; Oikonomopoulos-Zachos, C.; "Simulative Investigation of parameters Influencing the Reception Characteristics of Integrated Automobile Glass Antennas," POSTER, 2008. May 2008, Prague

[21] IEEE 802.15.4a model, final report, www.ieee.org

[22] Llano, G.; Reig, J.; Rubio, L.; , "Analytical Approach to Model the Fade Depth and the Fade Margin in UWB Channels," Vehicular Technology, IEEE Transactions on , vol.59, no.9, pp.4214-4221, Nov. 2010

[23] Sipal, V.; Allen, B.; Edwards, D.; , "Analysis and mitigation of antenna effects on wideband wireless channel," Electronics Letters, vol.46, no.16, pp.1159-1160, August 52010

[24] Sipal, V.; Allen, B.; Edwards, D.J.; " Effects of Antenna Impulse Response on Wideband Wireless Channel," Antennas \& Propagation Conference, 2010. LAPC 2010, November 2010, Loughborough 
Figures

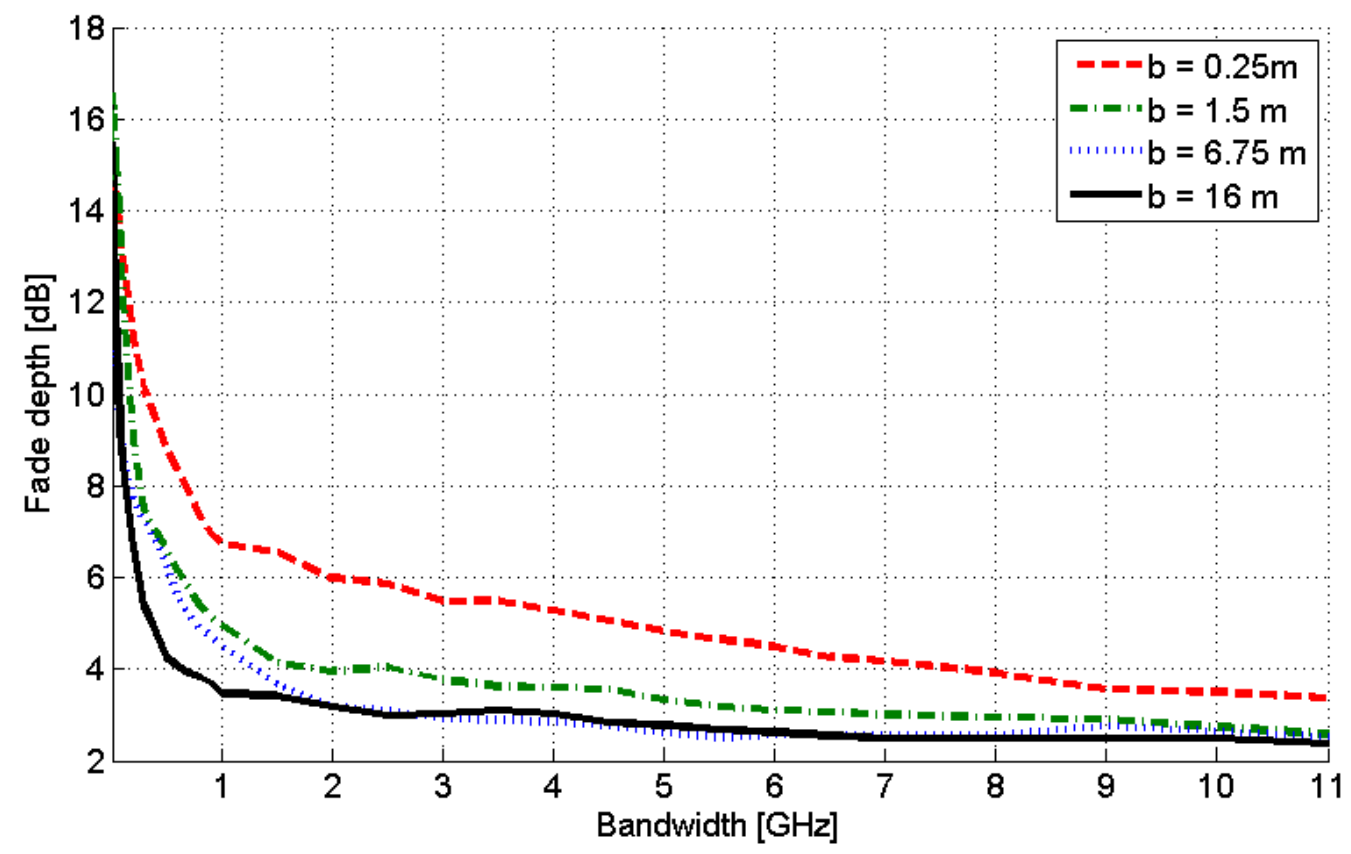

Fig.1 Fade depth scaling with variable bandwidth for varying room size
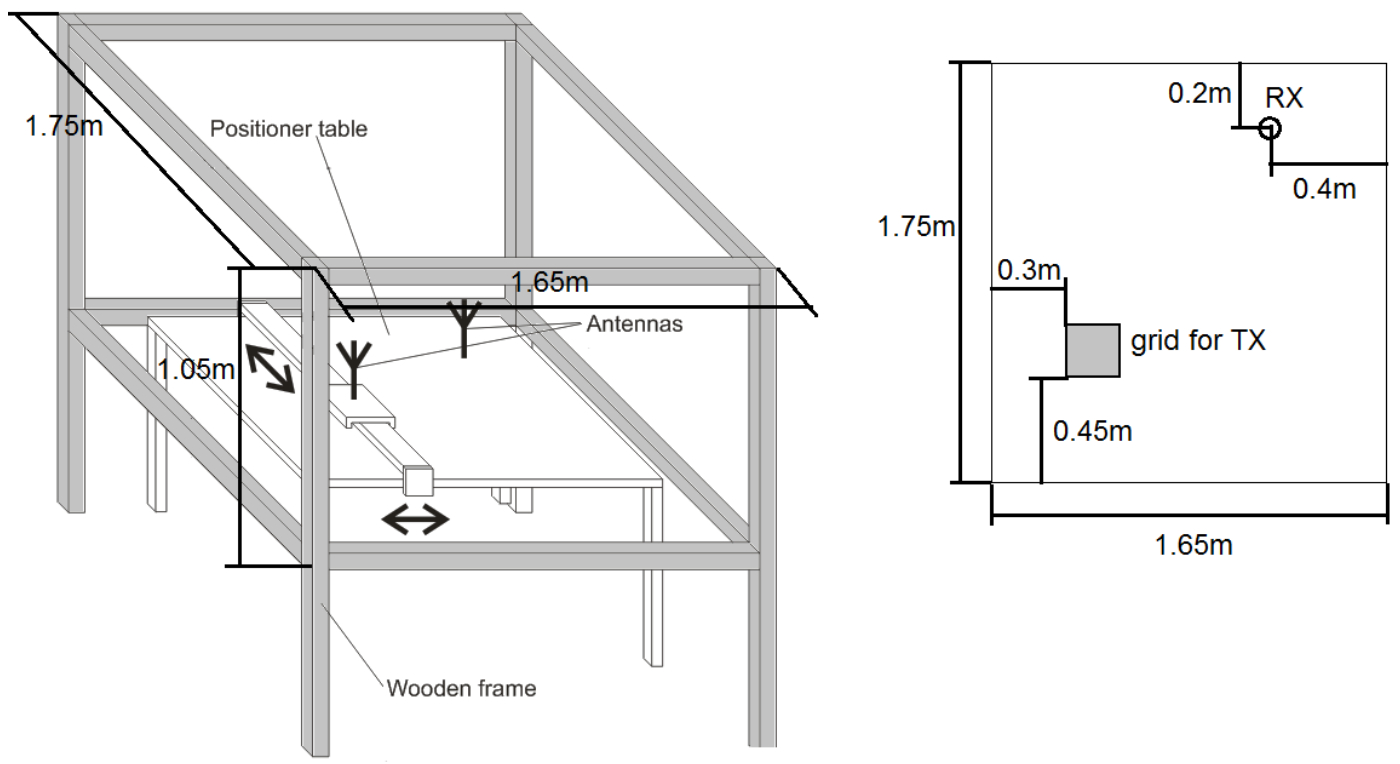

Fig. 2 Experimental confined environment with volume $3 \mathrm{~m}^{3}$ (roughly a car interior size), the right half of the figure represents a top view defining the antenna positions 


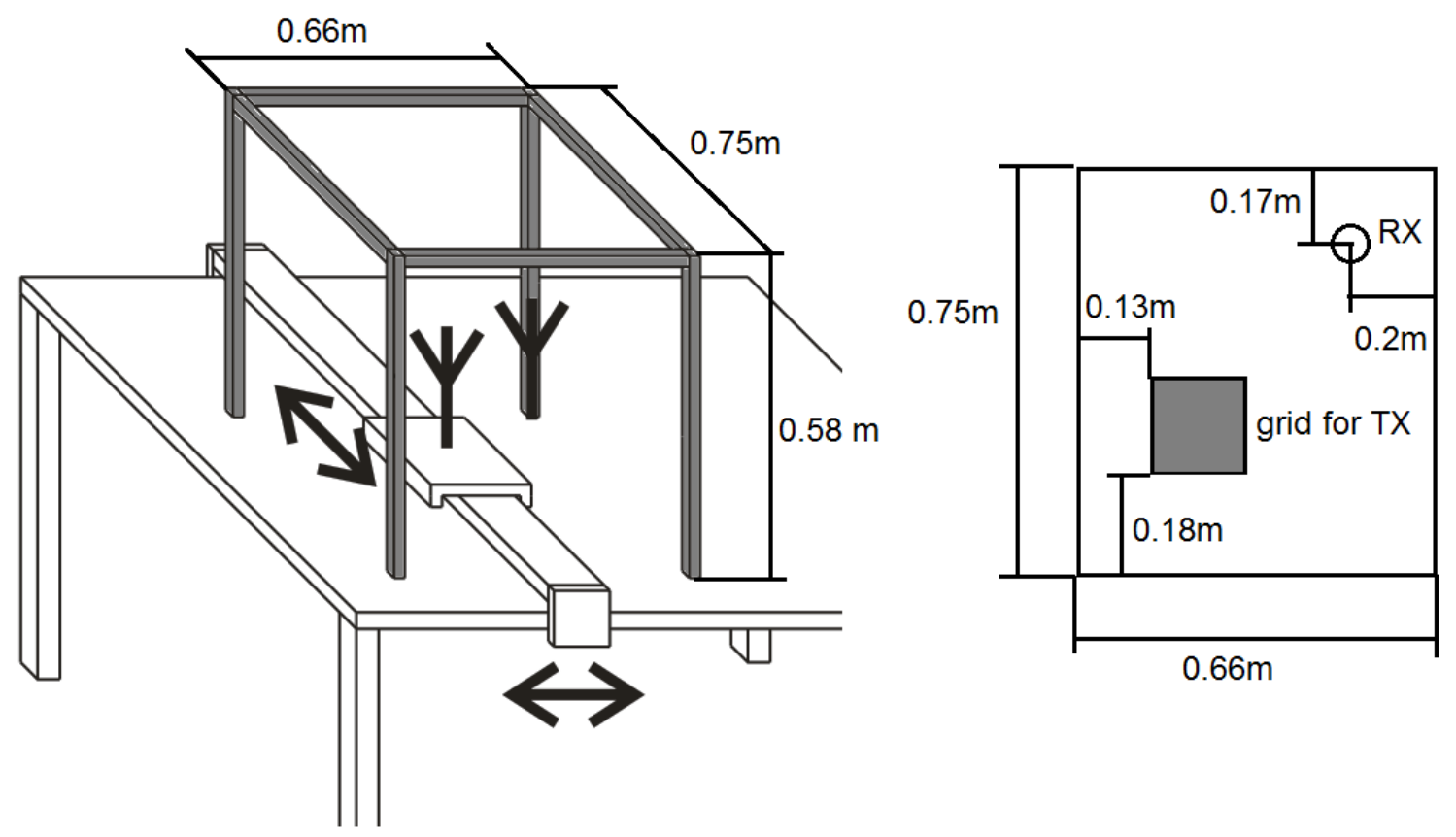

Fig. 3 Experimental confined environment with volume $0.3 \mathrm{~m}^{3}$, the right half of the figure represents a top view defining the antenna positions

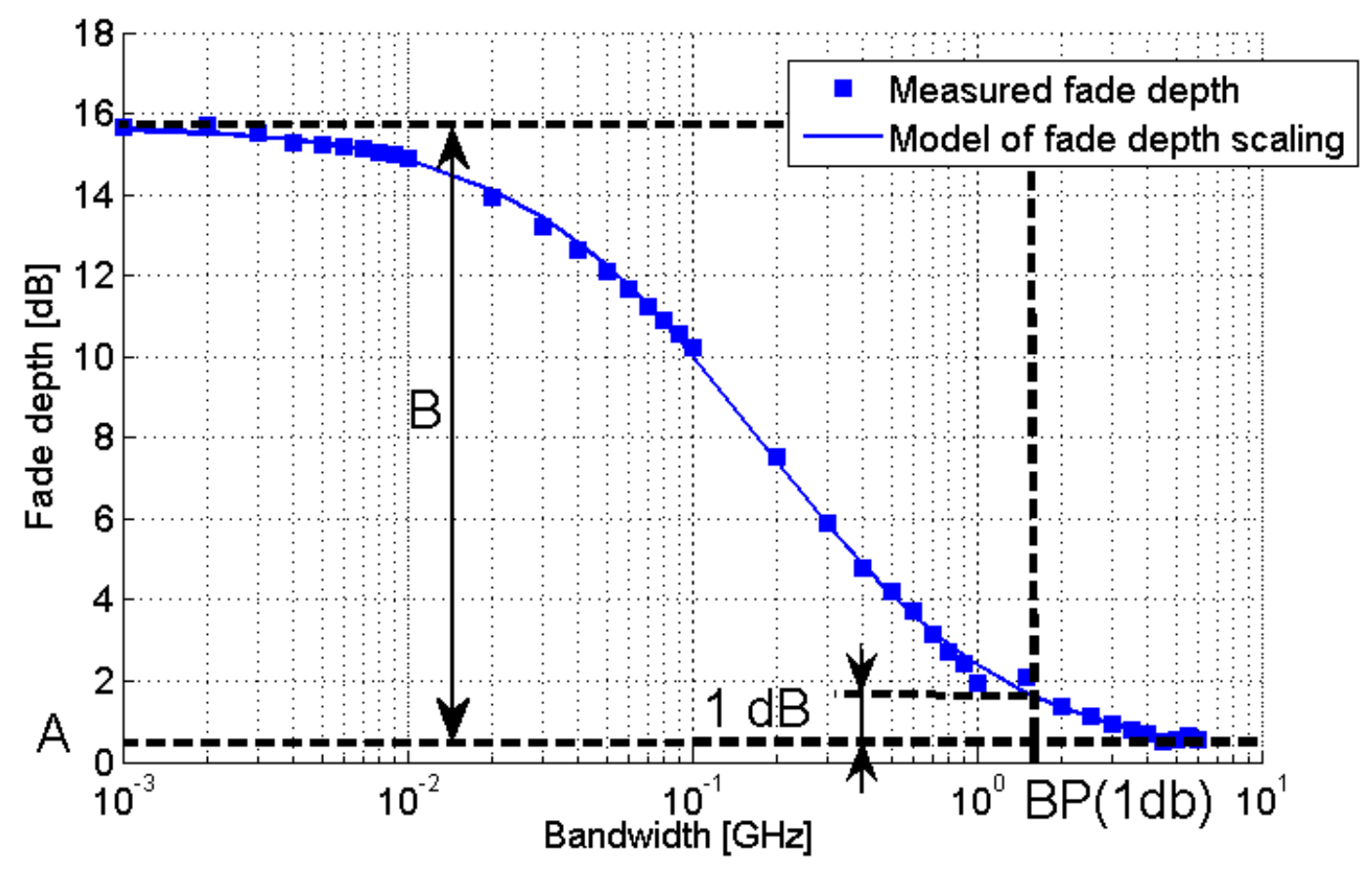

Fig. 4 Fade depth scaling model, the used data set is taken from [17] 


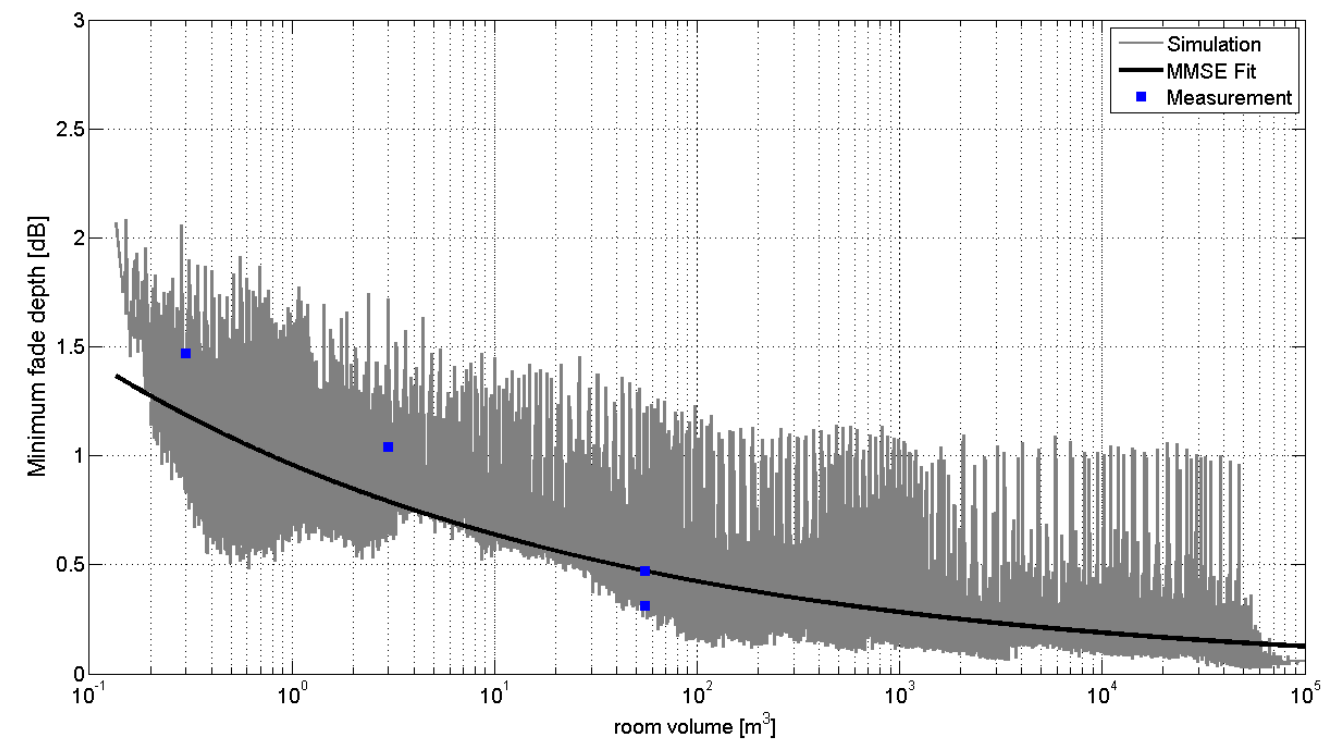

Fig. 5 Dependency of minimum fade depth on room volume

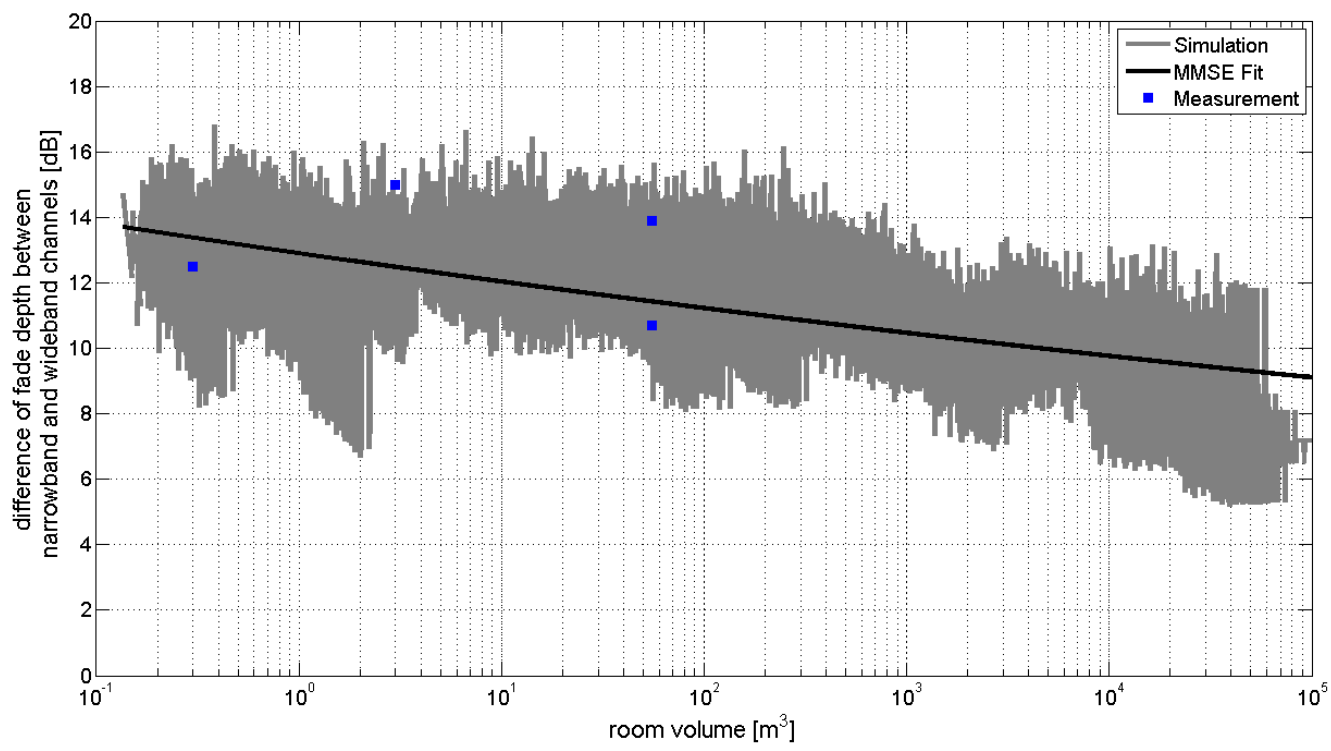

Fig. 6 Fade depth difference between narrowband and wideband channels in dependency on the room volume 


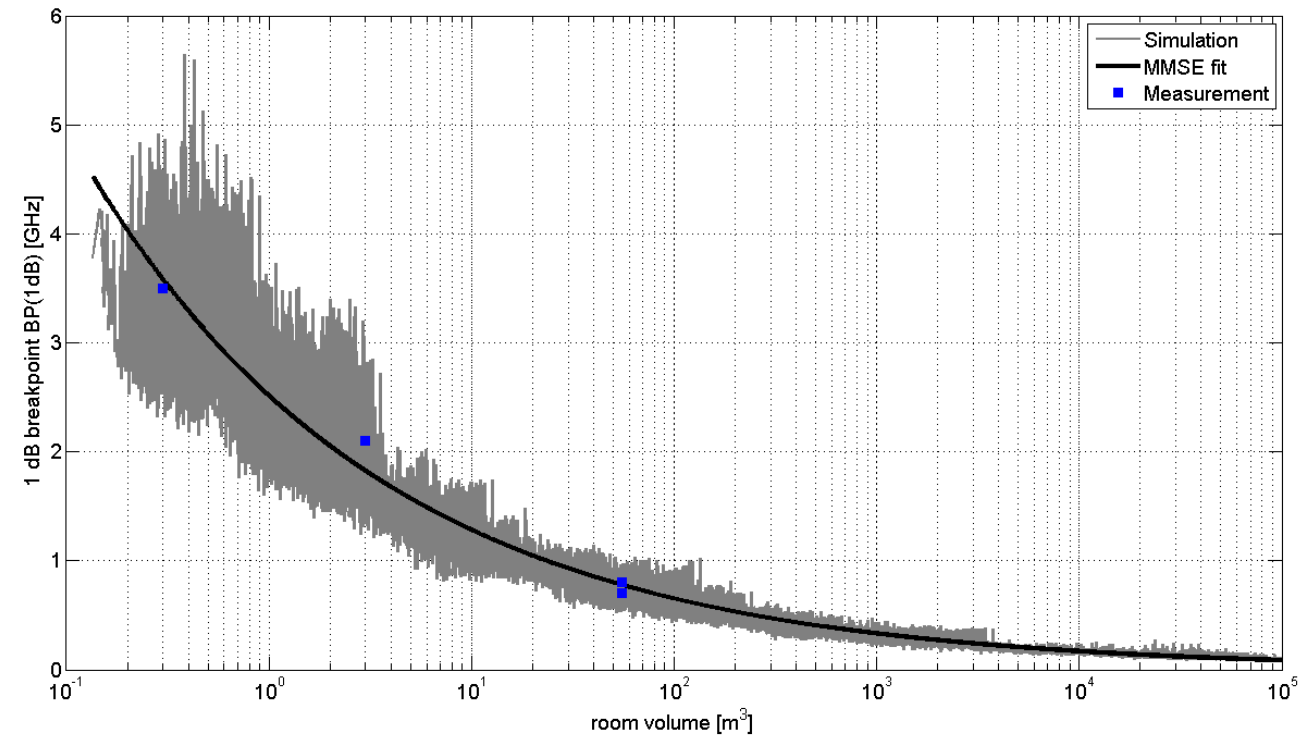

Fig. 7 Dependency of bandwidth break point $B P(1 \mathrm{~dB})$ on room volume 